\title{
The natural history of Wolff-Parkinson-White syndrome in 228 military aviators: A long-term follow-up of 22 years
}

Patrick J. Fitzsimmons, MD, a Paul D. McWhirter, MD, FACC,b David W. Peterson, MD, FACC,b and William B. Kruyer, MD, FACCa Brooks AFB and Lackland AFB, Tex

Background Wolff-Parkinson-White (WPW) syndrome poses a risk for tachyarrhythmias and sudden cardiac death. Most WPW studies have relatively limited numbers of subjects and brief follow-up periods.

Methods We reviewed records of 238 consecutive military aviators with WPW syndrome evaluated from 1955 to 1999. Follow-up was by questionnaires, telephone interviews, or death certificates. Events included sudden cardiac death and supraventricular tachycardia (SVT) (by electrocardiographic [ECG] documentation or suggestive symptoms).

Results The mean age was 34.3 years (range $17-56$ years). Forty-two $(42 / 238,17.6 \%$ ) had SVT (WPW syndrome) and 196 of $238(82.4 \%)$ had the WPW ECG pattern only. The mean follow-up of 21.8 years (range $2-41$ years) was obtained on 228 of $238(96 \%)$ for a total of 4906 patient-years. Sudden cardiac death occurred in 1 of 228 , an incidence of 0.0002 per patient-year (95\% confidence interval 0.0-0.001). SVT occurred in 47 of $228(20.6 \%)$ or 0.01 per patientyear. One hundred eighty-seven $(187 / 228,82 \%)$ initially had the WPW ECG pattern only; 28 of $187(15.0 \%)$ reported SVT during follow-up. Forty-one $(41 / 228,18 \%)$ initially had WPW syndrome; 19 of $41(46.3 \%)$ reported additional SVT during follow-up.

Conclusion Sudden cardiac death risk was low (0.02\%/patient-year) in this WPW population. The SVT incidence was $1 \%$ per patient-year. Referral bias and some characteristics of the unique military aviator population may partly account for these low event rates. However, these results may be more applicable to unselected populations than are tertiary referralbased studies. (Am Heart J 2001;142:530-6.)

Every year many individuals with no history of organic heart disease die from sudden cardiac death. Autopsy may reveal underlying pathologic conditions such as coronary artery disease or hypertrophic cardiomyopathy. However, there may be no apparent structural heart disease; one possible cause for death is a tachyarrhythmia involving an accessory pathway. ${ }^{1}$ The association of Wolff-Parkinson-White (WPW) syndrome and sudden cardiac death has been known since the 1930s. ${ }^{2}$ The mechanism is thought to be degeneration of supraventricular tachycardia (SVT) into atrial fibrillation, resulting in a rapid ventricular response over the accessory pathway and degeneration to ventricular fibrillation. ${ }^{3}$ Although management of individuals with frequent SVT, especially with asso-

From the aInternal Medicine Branch, Aeromedical Consultation Service, US Air Force School of Aerospace Medcine, Brooks AFB, and the bepartment of Cardiology, Wilford Hall US Air Force Medical Center, Lackland AFB, Tex.

The opinions and views expressed herein are those of the authors and do not necessarily represent the official views of the US Air Force or the Department of Defense. Submitted September 6, 2000; accepted June 7, 2001.

Reprint requests: William B. Kruyer, MD, USAFSAM/FECI 2507 Kennedy Circle, Brooks AFB, TX 78235-5117.

E-mail: william.kruyer@brooks.af.mil

4/1/117779

doi: 10.1067/mhi.2001.117779 ciated hemodynamic symptoms, is well defined, the treatment of individuals without such tachyarrhytmias is less clear. The literature reports that $12 \%$ to $27 \%$ of survivors of ventricular fibrillation and WPW had no prior tachyarrhythmias. ${ }^{4,5}$ Thus even individuals with only the WPW electrocardiographic (ECG) pattern are at risk for sudden cardiac death. However, the natural history of WPW for the development of SVT and sudden cardiac death has not been well defined. Several studies have examined the natural history of WPW for the development of arrhythmias and sudden cardiac death but most have had relatively small numbers and limited follow-up. 5-8

Although the prevalence of the WPW ECG pattern in the general population is estimated to be 1.0 to 3.0 per $1000,{ }^{9-13}$ the true prevalence is probably higher. Intermittent pre-excitation may not be diagnosed and many people do not routinely have an ECG. ${ }^{14}$ In a populationbased study, Munger et $\mathrm{al}^{8}$ reported that pre-excitation was absent on initial ECG in $22 \%$ of all patients who were later diagnosed with WPW syndrome. Intermittent pre-excitation may be related to the length of the accessory pathway effective refractory period, the location of the accessory pathway, or to variations in autonomic tone. ${ }^{15-17}$ Because of small reported numbers, the devel- 
opment of SVT in intermittent pre-excitation has not been explored.

Many tertiary care-based studies of WPW suffer from referral bias and may not reflect the true incidence of SVT and sudden cardiac death in a local community. ${ }^{9-13}$ The purpose of this study was to report the natural history of WPW in a nontertiary care population for the development of sudden cardiac death and SVT.

\section{Methods}

In the US Air Force a screening ECG is performed on all flying training applicants and also periodically on all trained aviators beginning at age 35 years. Training applicants found to have WPW syndrome are generally disqualified. Trained aviators with newly diagnosed WPW syndrome are referred to a central facility for evaluation and recommendation regarding continued flying status. We retrospectively reviewed records of 238 military aviators who were referred with WPW syndrome. To ensure the diagnosis of the WPW ECG pattern and determine intermittent versus continuous pre-excitation, an internist or cardiologist reviewed all ECGs of each subject. Subjects were separated into 2 categories: those with continuous pre-excitation and those with intermittent pre-excitation. To be classified as having intermittent pre-excitation, the subject had to have at least one ECG with no pre-excitation and a later ECG with pre-excitation consistent with WPW syndrome. Data collection began in 1955 and was completed in 1999. All individuals received comprehensive medical history and physical examination by an internist or cardiologist. Holter monitoring and graded exercise treadmill tests were performed as the technology became available. For the purpose of this study, initial presentation was defined as the combination of the local evaluation diagnosing WPW syndrome and the results of our initial central facility evaluation. After initial evaluation, individuals were divided into 2 groups: those with WPW syndrome (WPW ECG findings plus SVT by ECG documentation or by suggestive symptoms [rapid or racing heart sensation]) and those with WPW ECG pattern only (no documented or suspected SVT). ECG-documented SVT was defined as 3 or more consecutive nonsinus supraventricular complexes at a rate $\geq 100$ beats/min

Forty-five of the 238 subjects returned to the central facility for multiple occupational evaluations. These individuals had repeat noninvasive testing, and the data from these multiple evaluations were separated from data collected from the initial evaluations

Follow-up was conducted by questionnaire, telephone interview, medical records, and death certificate with 228 individuals completing follow-up requirements. These individuals were also separated into the 2 groups, WPW syndrome versus WPW ECG pattern only, on the basis of their initial presentations. End points were sudden death and SVT. These groups were further divided into patients who had intermittent or continuous pre-excitation. At the initial evaluation, associated cardiac diagnoses were also recorded.

\section{Statistical analysis}

Data analysis was performed on a VAX 6320 mainframe with a Smartstar structured query language addressing a Share- base 700 relational database. Statistical analysis was performed with use of programs from the SAS Institute ${ }^{18}$ on a VAX $11 / 780$ minicomputer. Results are reported as the mean \pm SD. The Cochran-Mantel-Haenzel method was used to estimate common relative risk. Calculations used to obtain the confidence limits were based on those described by Gail and Ware. ${ }^{19}$ Hypothesis testing methods to obtain confidence intervals were used as described by Kleinbaum et al. ${ }^{20}$

\section{Results \\ Initial presentation}

Demographics. The study cohort totaled 238 subjects who completed the initial evaluation, including $232(97 \%)$ men and $6(3 \%)$ women. The mean age at the initial evaluation was 34 years $( \pm 8.9$ years). The median age was 35 years and the range was 17 to 56 years. At the time of initial diagnosis, 88 patients were $<30$ years old, 96 patients were between 30 and 40 years old, and the remaining 54 patients were $>40$ years old. From review of prior ECGs, $49 \%$ had intermittent pre-excitation and $51 \%$ had continuous pre-excitation. Forty-two of 238 (17.6\%) initially had WPW syndrome. The age group with the highest prevalence of SVT was the 30- to 40-year age group. In this age group 21 of 96 (22\%) had SVT (WPW syndrome) at initial presentation. Nearly $15 \%$ of patients younger than age 30 years or older than age 40 years had WPW syndrome at the time of initial presentation. Age and presentation status are summarized in Figure 1.

WPW syndrome at initial presentation (Table I). Twenty-eight of the 238 (11.7\%) had SVT only by suggestive symptoms (racing heart sensation). Thirteen of these 28 had hemodynamic symptoms associated with their episodes of suspected SVT. One had syncope and the other 12 had presyncope, lightheadedness, dizziness, or dyspnea. Fourteen of the 238 (5.9\%) had ECGdocumented SVT, 6 on ECG rhythm strip, 4 on 24-hour Holter monitoring, and 4 on graded exercise treadmill testing. Three of these 14 subjects had hemodynamic symptoms associated with SVT.

Six of the 238 were seen in a local medical facility with complaints of palpitations or racing heart sensation and had SVT documented on an ECG rhythm strip. Two of the 6 also had associated hemodynamic symptoms. One had lightheadedness and the other had dyspnea and chest discomfort.

During the initial evaluation, 100 patients (100/238, $42 \%$ ) received a 24-hour Holter monitor. Four had SVT and one had a wide complex tachycardia felt to be ventricular tachycardia rather than antidromic SVT. None of the patients had hemodynamic symptoms associated with Holter monitor-documented arrhythmia. Other Holter monitor findings are summarized in Table II. The most common Holter monitor finding was rare premature atrial contractions followed by rare multiform premature ventricular contractions. 


\section{Figure 1}

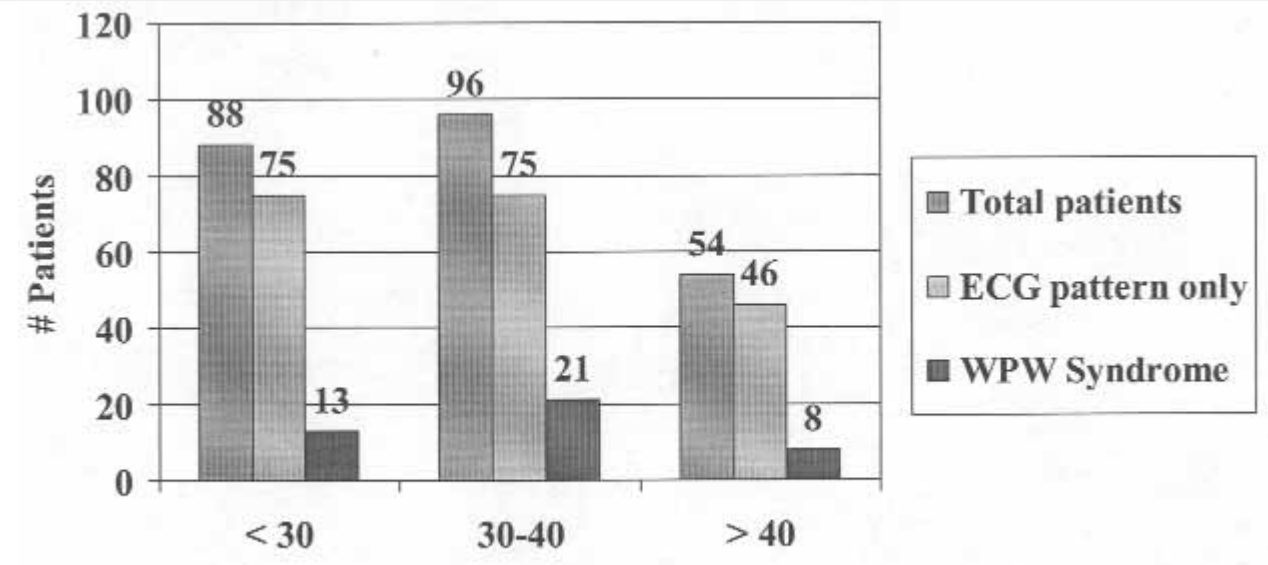

Age (Range 17-56)

Age and status at initial presentation.

Table I. Documented arrhythmias on noninvasive testing

\begin{tabular}{lccc}
$\begin{array}{l}\text { Noninvasive } \\
\text { test }\end{array}$ & $\begin{array}{c}\text { Heart } \\
\text { rate } \\
\text { (beats/min) }\end{array}$ & $\begin{array}{c}\text { Duration } \\
\text { (min) }\end{array}$ & Arrhythmia \\
\hline $\begin{array}{l}\text { Initial evaluation } \\
\text { Rhythm strip }\end{array}$ & $150^{*}$ & 7 & A flutter \\
Rhythm strip & $250^{*}$ & 7 & SVT \\
GXT & $220^{*}$ & 1 & SVT \\
Rhythm strip & 167 & 4 & SVT \\
Rhythm strip & 168 & 5 & SVT \\
Rhythm strip & 180 & 4 & SVT \\
Rhythm strip & 230 & 5 & SVT \\
HM & 126 & 3 & SVT \\
HM & 130 & 5 & SVT \\
HM & 185 & 1 & SVT \\
HM & 210 & 7 & SVT \\
HM & 300 & $<1(10$ s) & VT \\
GXT & 180 & 9 & SVT \\
GXT & 200 & 8 & SVT \\
GXT & 220 & 5 & SVT \\
Follow-up evaluation & & & \\
GXT & 160 & $<1(12$ beats) & SVT \\
GXT & 210 & 9 & SVT \\
HM & 177 & $<1(3$ beats) & SVT \\
HM & 150 & $<1(3$ beats) & SVT \\
\hline
\end{tabular}

GXT, Graded exercise test; HM, Holter monitor; VT, ventricular tachycardia. *Hemodynamic symptoms (syncope, presyncope, lightheadedness, dizziness, dyspnea).

Graded exercise treadmill testing was performed on 228 of 238 (96\%) patients during initial evaluation. Four had SVT. One patient with SVT on graded exercise treadmill testing had associated hemodynamic symptoms. He had SVT for nearly a minute at a rate of 220 beats/min with associated racing heart sensation, presyncope, dyspnea, and chest discomfort.
In summary, at initial evaluation, 15 patients had a documented tachyarrhythmia (14 SVT, 1 ventricular tachycardia) on ECG rhythm strip or noninvasive testing. Three (all with SVT) had symptoms suggesting hemodynamic compromise. These arrhythmias are summarized in Table I. Tachyarrhythmia on noninvasive testing did not predict patients who had additional SVT during the follow-up period.

Additional diagnoses at initial presentation. Physical examination and echocardiography revealed 3 individuals with mitral valve prolapse, 1 with hypertrophic cardiomyopathy, and 1 with mild aortic insufficiency and an otherwise normal 3-leaflet aortic valve. One patient had the concomitant diagnosis of left bundle branch block on ECG. Thirteen subjects underwent occupational coronary angiography for abnormal noninvasive testing. Six (46\%) of these 13 had asymptomatic, significant coronary artery disease (single lesion $\geq 50 \%$ luminal diameter reduction), and 3 had nonocclusive coronary artery disease. Three subjects had SVT (WPW syndrome) at the time of initial presentation. Two of the subjects with mitral valve prolapse and the subject with mild aortic insufficiency had ECG-documented SVT; none of these 3 had associated hemodynamic symptoms. During follow-up, there was no SVT in any of these subjects with additional cardiac diagnoses at initial presentation.

\section{Follow-up}

Demographics. Follow-up was obtained in 228 (96\%) of 238 individuals. The mean follow-up period was 21.8 years ( \pm 11.7 years) with a range of 2 to 41 years for a total of 4906 patient-years.

Mortality. There were 34 total deaths; 21 were noncardiac (17 medical illness, 4 traumatic), 12 were due 
to atherosclerotic coronary artery disease, and one was due to sudden cardiac death associated with WPW syndrome. The incidence of sudden death associated with WPW was 0.0002 per patient-year ( $95 \%$ confidence interval 0.0-0.001).

The mean age of the 17 patients with noncardiac medical illness deaths was 60.9 years (range 50-79 years). Three of these 17 subjects had WPW syndrome before death. The 4 traumatic deaths included 2 resulting from motor vehicle accidents and 2 suicides. These 4 subjects all had WPW ECG pattern only and the 2 motor vehicle accident subjects had intermittent pre-excitation.

None of the 12 patients who died from atherosclerotic coronary artery disease received coronary angiography during their initial evaluations. The mean age at death was 67.6 years (range 57-80 years). Only 1 of these 12 deaths was sudden and occurred in the setting of an acute myocardial infarction at age 61 years; this individual had only intermittent pre-excitation and WPW ECG pattern only. Only 1 of these 12 patients had WPW syndrome; he died at the age of 75 years.

The one patient who, in our opinion, had WPWassociated sudden cardiac death was initially seen by us with WPW syndrome and continuous pre-excitation. He had no family history of sudden cardiac death. We were able to obtain only limited medical records from the family. During follow-up he reportedly had recurrent SVT and atrial fibrillation and was placed on metoprolol and encainide. At the age of 60 years, he had an episode of atrial fibrillation with rapid ventricular response and was hospitalized with a diagnosis of congestive heart failure. Later during that hospitalization, coronary angiography demonstrated normal coronary arteries, according to the patient's later report. No information was available to us regarding left ventricular function. After the hospitalization, encainide was discontinued, and metoprolol was changed to propranolol. While he was taking propranolol, the ECG no longer showed evidence of pre-excitation. He reportedly was seen in an emergency department 18 months later with an unspecified tachyarrhythmia that quickly decompensated into cardiopulmonary arrest. Resuscitative efforts were unsuccessful and the patient died within 90 minutes of symptom onset. From the limited records available to us, it appeared that his dyspneic symptoms (diagnosed as "congestive heart failure") occurred only during tachyarrhythmias. In one earlier outpatient note, the patient reported direct current cardioversion at his home by emergency medical technicians for a heart rate in the 300s. Additionally, 2 ECGs dated 2 and 3 days before his death were available for our review. Both clearly demonstrated atrial fibrillation with rapid ventricular response and pre-excitation. It appeared therefore that his death a few days later was likely due to atrial fibrillation with rapid ventricular response and degeneration to ventricular fibrillation. Although his pattern of preexcitation resolved on propranolol therapy, we classified this patient as having a continuous WPW syndrome because of his initial presentation and persistent WPW pattern off treatment.

SVT. Overall, 47 of 228 (20.6\%) had SVT (first or recurrent episode) during the follow-up period. The occurrence of SVT during follow-up is displayed in Figure 2.

Forty-one (18\%) of the 228 patients initially had WPW syndrome. Nineteen $(46.3 \%)$ of these 41 patients reported additional SVT during the follow-up period and 8 of the 19 reported associated hemodynamic symptoms. Eleven of the 19 reported only one episode of SVT during follow-up and 8 of the 19 reported recurrent episodes of SVT ( 7 with $\leq 2$ episodes per year and 1 with $>2$ episodes per year). Of these 19 subjects who were seen initially with WPW syndrome and who had additional SVT during follow-up, 10 had continuous pre-excitation at the initial evaluation.

One hundred eighty-seven patients were initially seen with the WPW ECG pattern only. A first episode of SVT occurred in 28 (15.3\%) of these 187 patients during follow-up. Four of these 28 subjects had their first episodes of SVT on follow-up noninvasive testing (Table I) at our central facility; the other 24 reported their first episodes on the follow-up questionnaire. Nineteen of these 28 subjects reported associated hemodynamic symptoms. Fourteen of the 28 reported only one episode of SVT during follow-up and the other 14 reported recurrent episodes of SVT ( 5 with $\leq 2$ episodes per year and 9 with $>2$ episodes per year). Nineteen of these 28 subjects who later had WPW syndrome had continuous pre-excitation at their initial evaluations.

Thus 19 (23\%) of the 84 patients who initially had the WPW ECG pattern only and with continuous preexcitation had SVT during follow-up. Only 9 (8.7\%) of the 103 patients who initially had the WPW ECG pattern only and intermittent pre-excitation had SVT during follow-up. Although there was no statistical difference between patients with WPW ECG pattern only and continuous or intermittent pre-excitation regarding the occurrence of the first episode of SVT, a higher percentage of patients with continuous pre-excitation (23\% vs $8.7 \%$ ) had SVT during follow-up.

The overall event rate for SVT was $1.0 \%$ per patientyear. The 41 patients who initially had WPW syndrome had additional SVT at a rate of only $2.0 \%$ per year. The 187 patients who initially had WPW ECG pattern only had a first episode of SVT at an event rate of $0.7 \%$ per patient-year.

Invasive cardiac testing and antiarrhythmic therapy. Only 16 of the 228 (7\%) underwent electrophysiologic testing during follow-up. The indications included 8 patients with SVT, 4 with syncope, 1 with congestive heart failure (from chronic atrial fibrillation and uncontrolled ventricular response), and 3 with WPW ECG 


\section{Figure 2}

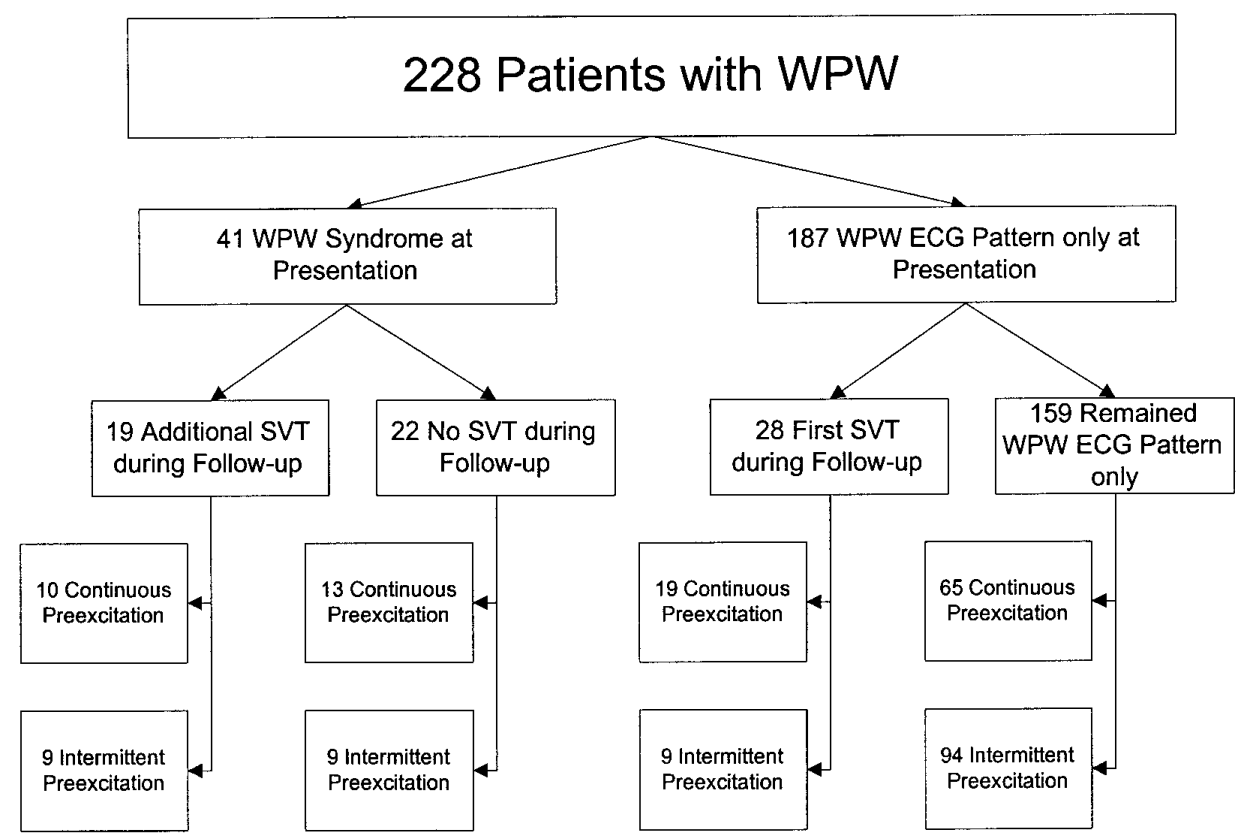

Long-term follow-up for suspected or ECG-documented SVT in 228 patients with WPW syndrome.

Table II. Spectrum of other Holter monitor findings in patients with WPW syndrome $(n=100)$ during initial evaluation

\begin{tabular}{lccc} 
& Rare & Occasional & Frequent \\
\hline PAC & 30 & 2 & 0 \\
Uniform PVC & 24 & 3 & 3 \\
Multiform PVC & 3 & 1 & 0 \\
PJC & 2 & 1 & 1 \\
\hline
\end{tabular}

PAC pair 5, wandering atrial pacer 3. Rare ectopy was defined as $\leq 0.1 \%$ of total beats, occasional as $>0.1 \%$ and $\leq 1.0 \%$, frequent as $>1.0 \%$ and $\leq 10 \%$, and very frequent as $>10 \%$ of total beats (no subjects had very frequent ectopy). PAC, Premature atrial contraction; PVC, premature ventricular contraction; PJC, premature junctional contraction.

pattern only for elective ablation. Sixteen of the 228 patients were placed on antiarrhythmic therapy. Referral for electrophysiologic testing was low, but most of the follow-up period was before the current technologic development of electrophysiologic testing and radiofrequency ablation as curative therapy.

\section{Discussion}

\section{Sudden cardiac death}

Previous natural history studies have reported sudden cardiac death rates of $0.0 \%$ to $0.6 \%$ per year for patients with WPW. ${ }^{21-24}$ Our study included patients with both WPW syndrome and WPW ECG pattern only, and 49\% of our subjects had only intermittent pre-excitation. There was only one definite WPW associated with sudden death. There was one other sudden cardiac death, occurring in the setting of acute myocardial infarction, and there were 2 automobile accident deaths. All 3 of these individuals had intermittent pre-excitation. It is therefore very unlikely that WPW was related to their deaths. Our sudden cardiac death incidence of $0.02 \%$ per year was similar to other natural history studies, as summarized in Table III. ${ }^{5-8}$ Generally, patients with intermittent pre-excitation are thought to have an accessory pathway with conduction characteristics that provide a lower risk for sudden cardiac death during atrial fibrillation. Electrophysiologic testing has demonstrated that antegrade conduction through these accessory pathways is poor. These patients cannot therefore propagate a rapid ventricular response during atrial fibrillation, conferring a lower risk of sudden cardiac death. ${ }^{25}$ With only one WPW-associated sudden cardiac death, our study is underpowered to address this risk in patients with intermittent preexcitation.

\section{SVT}

For the purpose of this study we included individuals with any SVT, including short runs of asymptomatic ECG-documented SVT. The clinical significance of nonsustained SVT in WPW is not defined. Our institution is concerned about any SVT as a potential risk for sudden incapacitation during military flight. This combined with 
Table III. Summary of natural history studies of WPW syndrome

\begin{tabular}{|c|c|c|c|c|c|}
\hline & \multicolumn{5}{|c|}{ Author } \\
\hline & Leitch et al5 & Guize et al6 & $\begin{array}{l}\text { Berkman and } \\
\text { Lamb }\end{array}$ & Munger et al8 & Current study \\
\hline Years & 1980-1988 & $1971-1980$ & $1933-1961$ & 1953-1989 & $1955-1999$ \\
\hline No. of patients & 75 & 170 & 128 & 113 & 228 \\
\hline Total patient-years follow-up & 348 & 695 & 1157 & 1338 & 4906 \\
\hline Average years of follow-up & 4.3 & 4.6 & 9 & 11.8 & 21.8 \\
\hline Age range $(y)$ & $7-77$ & $20-70$ & $25-40$ & $0-77$ & $17-56$ \\
\hline Percent follow-up & 100 & 89 & 100 & 95 & 96 \\
\hline No. with sudden cardiac death & 0 & 1 & $0-1$ & 2 & 1 \\
\hline Sudden cardiac deaths per year & 0.000 & 0.0014 & $0.000-0.0009$ & 0.0015 & 0.0002 \\
\hline SVT (per patient-y) & $1.7 \%$ & NA & $1.5 \%$ & $3 \%$ & $1 \%$ \\
\hline
\end{tabular}

NA, Not available.

the lack of a standard definition for sustained SVT led us to include all SVT for data analysis. However, there were only 3 individuals with clearly nonsustained SVT (12 beats or less duration, Table I). These 3 all presented initially with WPW ECG pattern only and had nonsustained SVT recorded on follow-up noninvasive testing. None of the 3 reported any other SVT during the follow-up period. Thus this inclusion had little impact on the results. Removing these 3 from consideration would further reduce the low $(0.7 \% / y)$ incidence of SVT in those seen initially with WPW ECG pattern only. However, this small number of clearly nonsustained SVT allowed no conclusions about the significance of ECG-documented nonsustained SVT in WPW syndrome.

Approximately 20\% of our patients had SVT (first or recurrent episode) during a mean follow-up over 20 years. Although electrophysiologic studies have demonstrated weak antegrade conduction down the accessory pathway in patients with intermittent preexcitation, thus minimizing the likelihood of sudden death, retrograde conduction up the accessory pathway may be quite functional and able to propagate orthodromic re-entrant SVT. But the development of SVT in these patients may be less frequent because of pathway location, longer refractory periods, or vagal tone. Debate remains whether some intermittent or continuous pathways become nonfunctional over time, reducing the incidence of SVT with advancing age. This report argues against prior natural history studies that have suggested a benign course for asymptomatic patients with no family history for sudden cardiac death who present at $>40$ years of age. ${ }^{14}$ In our study, $11 \%$ of WPW ECG pattern, only subjects that initially presented older than age 40 years later had WPW syndrome. None of these patients had a family history for sudden cardiac death. These patients may not be at risk for sudden cardiac death, but all patients appear to be at risk for SVT regardless of age at initial diagnosis. Noninvasive testing did not predict patients who were at risk for development of SVT (first or recurrent episode) or sudden cardiac death during the follow-up period.

\section{Study limitations}

There are several limitations to this study. The largest limitation is selection bias regarding the reporting of SVT. Before radiofrequency ablation, US Air Force aviators with WPW syndrome were permanently disqualified from flying. Therefore many aviators who presented to our central facility may have underreported symptoms suggesting SVT and some with ECG documented SVT may not have even been referred. Reporting of SVT during follow-up is more accurate because most patients were retired or no longer required qualification for flying status to continue their current career. And our annual event rate of $1.0 \%$ per year for SVT is comparable to other natural history studies, ${ }^{, 7,8}$ as shown in Table III.

A second limitation is the large percentage of individuals with intermittent WPW. Prior studies have suggested that $20 \%$ to $60 \%$ of patients have intermittent pre-excitation. ${ }^{14,26}$ US Air Force pilots typically have a screening ECG before entry into undergraduate pilot training. Applicants with WPW syndrome or with WPW ECG pattern only are typically disqualified from entering into undergraduate pilot training. However, if WPW syndrome is initially diagnosed in a trained aviator, continued flying may be allowed for those with WPW ECG pattern only. All pilots on flying status receive serial screening ECGs beginning at age 35 years. Thus many may have a normal ECG in their early 20s on applying for flying training and later have an ECG with a WPW pattern of pre-excitation at age 35 years or older. The large number of intermittent WPW patterns allows comparison with continuous pre-excitation but may cause the risk for sudden cardiac death to be lower than other studies.

Finally, the demographics are concentrated around 
relatively young men. There were only 4 female patients and no children in the study. With the absence of children who may seek medical attention at a young age, the study group may imply a lower rate of symptoms and sudden cardiac death, a survivor bias. Because of the age range of military service, this study does not provide event rates in older age groups of patients with WPW syndrome. And the Air Force has had female pilots for only a relatively short time.

Despite these limitations, this study provides important information for the most frequently diagnosed age groups of patients with WPW syndrome. This study reveals lower event rates for sudden cardiac death and SVT than other tertiary care referral-based studies and may more accurately reflect the natural history of WPW syndrome in the general population. The large percentage of patients with intermittent pre-excitation suggests that other studies may underestimate the true prevalence of WPW syndrome.

\section{Conclusion}

The risk of sudden cardiac death appears to be low and may be more likely to occur in patients with WPW syndrome with continuous pre-excitation. The incidence of a first episode of SVT in those presenting initially with the WPW ECG pattern only was $0.7 \%$ per patient-year, and subjects initially presenting with WPW syndrome had recurrent SVT at a rate of only 2\% per patient year. However, the cumulative lifetime risk of SVT might be considered clinically significant. Noninvasive testing did not predict the development of SVT (first or recurrent episode) or sudden cardiac death. SVT appears to occur more frequently in patients with continuous pre-excitation than in patients with intermittent pre-excitation and it can occur at any age. Sudden cardiac death can also occur at any age but is likely a risk only in patients with continuous pre-excitation. Patients who are diagnosed over the age of 40 years should be treated as aggressively as their younger counterparts. The occurrence of SVT in all age groups requires regular medical follow-up.

We thank Mr Al Adair and Mr Bill Besich for computer assistance and database development. Special thanks go to Mr Bill Thompson for data analysis and statistical support.

\section{References}

1. Prystowsky EN, Fananapazir L, Packer DL. Wolff-Parkinson-White syndrome and sudden cardiac death. Cardiology 1987;74(2 Suppl):67-71.

2. Wood FC, Wolferth CC, Geckeler GD. Histologic demonstration of accessory muscular connections between auricle and ventricle in a case of short P-R interval and prolonged QRS complex. Am Heart J 1943;25:454-62.

3. Klein GJ, Bashore TM, Sellers TD. Ventricular fibrillation in the
Wolff-Parkinson-White syndrome. N Engl J Med 1979;301: 1080-5.

4. Montoya PT, Brugada P, Smeets J. Ventricular fibrillation in the WolffParkinson-White syndrome. Eur Heart J 1991;12:144-50.

5. Leitch JW, Klein GJ, Yee R. Prognostic value of electrophysiology testing in asymptomatic patients with Wolff-Parkinson-White pattern. Circulation 1990;82:1718-23.

6. Guize L, Soria R, Chaouat JC. Prevalence et evolution du syndrome de Wolff-Parkinson-White dans une population de 138,048 sujets. Ann Med Interne (Paris) 1985; 136:474-8.

7. Berkman NL, Lamb LE. The Wolff-Parkinson-White electrocardiogram: a follow-up study of five to twenty-eight years. N Engl J Med 1968;278:492-4.

8. Munger TM, Packer DL, Hammill SC. A population study of the natural history of Wolff-Parkinson-White syndrome in Olmsted County, Minnesota, 1953-1989. Circulation 1993;87:866-73.

9. Hiss RG, Lamb LE. Electrocardiographic findings in 122,043 individuals. Circulation 1962;25:947-61.

10. Sears GA, Manning GW. The Wolff-Parkinson-White pattern in routine electrocardiography. Can Med Assoc J 1962;87:1213-7.

11. Averill KH, Fosmoe RJ, Lamb LE. Electrocardiographic findings in 67,375 asymptomatic subjects, IV: Wolff-Parkinson-White syndrome. Am J Cardiol 1960;6:108-29.

12. Smith RF. The Wolff-Parkinson-White syndrome as an aviation risk. Circulation 1964;29:672-9.

13. Zipes DP, Ritchie JL. Guidelines for clinical intracardiac electrophysiological and catheter ablation procedures: a report of the American College of Cardiology/American Heart Association task force on practice guidelines (Committee on Clinical Intracardiac Electrophysiologic and Catheter Ablation Procedures) developed in collaboration with the North American Society of Pacing and Electrophysiology. J Am Coll Cardiol 1995;26:555-73.

14. Krahn AD, Manfreda J, Tate RB. The natural history of electrocardiographic preexcitation in men: the Manitoba follow-up study. Ann Intern Med 1992;1 16:456-60.

15. Prystowsky EN. Diagnosis and management of the preexcitation syndromes. Curr Probl Cardiol 1988;13:225-310.

16. Cain ME, Luke RA, Lindsay BD. Diagnosis and localization of accessory pathways. Pacing Clin Electrophysiol 1992;15:801-24.

17. Wellens HJ, Atie J, Penn OC. Diagnosis and treatment of patients with accessory pathways. Cardiol Clin 1990;8:503-21.

18. SAS Institute. SAS procedures guide, version 6. 3rd ed. Cary (NC): SAS Institute; 1990.

19. Gail M, Ware J. Comparing observed life table data with known survival curve in the presence of random censorship. Biometrics 1979;35:385-91.

20. Kleinbaum D, Kupper L, Morganstern H. Epidemiologic research: principles and quantitative methods. New York: John Wiley; 1982.

21. Kimball JL, Burch G. The prognosis of the Wolff-Parkinson-White syndrome. Ann Intern Med 1947;27:239-42

22. Klein GJ, Prystowsky EN, Yee R. Asymptomatic Wolff- ParkinsonWhite: should we intervene? Circulation 1989;80:1902-5.

23. Flensted-Jensen E. Wolff-Parkinson-White syndrome: a long-term follow-up of 47 cases. Acta Med Scand 1969; 186:65-74.

24. Gillette PC, Garson A Jr, McNamara DG. Wolff-Parkinson-White syndrome in children: natural history and clinical spectrum [abstract]. Circulation 1980;62(III Suppl):III271.

25. Pietersen $A H$, Andersen ED, Sandoe E. Atrial fibrillation in the Wolff-Parkinson-White syndrome. Am J Cardiol 1992;70:38-43A

26. Klein GJ, Gulamhusein SS. Intermittent preexcitation in the WolffParkinson-White syndrome. Am J Cardiol 1983;52:292-6. 